\title{
Photonic quasicrystals for general purpose nonlinear optical frequency conversion
}

\author{
Ron Lifshitz* \\ School of Physics and Astronomy, Raymond and Beverly Sackler \\ Faculty of Exact Sciences, Tel Aviv University, Tel Aviv 69978, Israel \\ Ady Arie and Alon Bahabad \\ School of Electrical Engineering, Wolfson Faculty of Engineering, Tel Aviv University, Tel Aviv 69978, Israel
}

(Dated: December 3, 2004)

\begin{abstract}
We present a general method for the design of 2-dimensional nonlinear photonic quasicrystals that can be utilized for the simultaneous phase-matching of arbitrary optical frequency-conversion processes. The proposed scheme-based on the generalized dual-grid method that is used for constructing tiling models of quasicrystals - gives complete design flexibility, removing any constraints imposed by previous approaches. As an example we demonstrate the design of a color fan - a nonlinear photonic quasicrystal whose input is a single wave at frequency $\omega$ and whose output consists of the second, third, and fourth harmonics of $\omega$, each in a different spatial direction.
\end{abstract}

PACS numbers: 42.65.Ky, 42.70.Mp, 61.44.Br, 42.79.Nv

The problem of phase matching in the interaction of light waves in nonlinear dielectrics became immediately evident as the first theories describing such interaction were developed [1]. Put simply, nonlinear interaction is severely constrained in dispersive materials because the interacting photons must conserve their total energy and momentum. Even the slightest wave-vector mismatch appears as an oscillating phase that averages out the outgoing waves, hence the term "phase mismatch". One approach for treating the problem uses the birefringent properties of specific materials and by playing with the polarizations of the interacting waves achieves phase matching 2, 3. . A second approach, suggested over 4 decades ago [1, 4] and known today as "quasi-phasematching", is to modulate the sign of the relevant component(s) of the nonlinear dielectric tensor at the period of the oscillating mismatched phase thereby undoing the averaging. Quasi-phase-matching has been generalized from simple 1-dimensional periodic modulation [5] to 2dimensional periodic modulation $[6,7,8,9,10$ as well as 1-dimensional quasiperiodic modulation [11, 12, 13, 14], allowing greater flexibility in phase-matching multiple frequency-conversion processes within the same photonic crystal. Here we present the full generalization of the method that enables the design of nonlinear photonic crystals that can simultaneously phase-match any arbitrary set of frequency-conversion processes in any spatial direction. This design flexibility is ideal for the realization of elaborate multi-step cascading effects [15, 16], as demonstrated by the color fan example (Fig. 1) at the end of this article.

To understand how the method works it is convenient to adopt the view taken in condensed matter systems. Recall that momentum conservation is a direct consequence of having continuous translation symmetry. In crystals, whether periodic or not, continuous translation symmetry is broken, and momentum conservation is re-

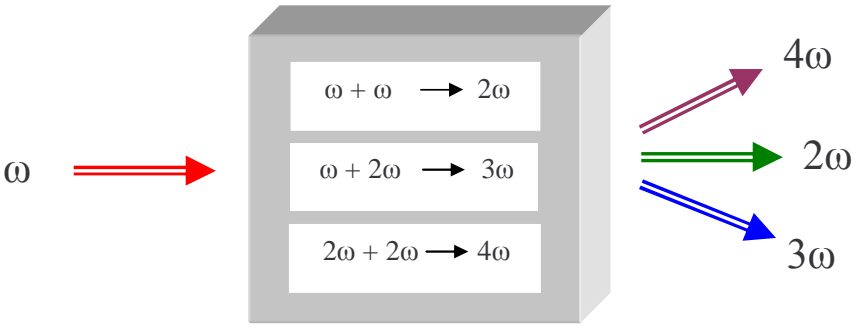

FIG. 1: The color fan.

placed by the less-restrictive conservation law of crystalmomentum. The total momentum of any set of interacting particles in a crystal - whether they are electrons, phonons, or photons - need only be conserved to within a wave vector from the reciprocal lattice of the crystal, giving rise to so-called umklapp processes. Thus, all one needs to do is to construct an artificial photonic crystal whose nonlinear susceptibility is ordered on the appropriate length scale such that its reciprocal lattice contains all the necessary wave vectors, needed to phase-match any required frequency-conversion process. The linear susceptibility should remain constant in space so that the dispersion relation will not vary from point to point. We show here a method - borrowed from the theory of quasicrystals [17] - to construct ordered structures in real space with any given Fourier components. Such structures are in general quasiperiodic, thus we are concerned here with the design of nonlinear photonic quasicrystals (for a definition of the term see the discussion in Ref. 18).

To be more specific, consider for example, two incoming waves $\left(\omega_{1}, \mathbf{k}_{1}\right)$ and $\left(\omega_{2}, \mathbf{k}_{2}\right)$ interacting in the plane of a 2-dimensional dielectric whose total area is $A$, via its lowest-order nonlinear susceptibility tensor $\chi^{(2)}$, to produce an outgoing wave $\left(\omega_{3}, \mathbf{k}_{3}\right)$, with $\omega_{3}=\omega_{1}+\omega_{2}$ and a wave vector mismatch $\Delta \mathbf{k}=\mathbf{k}_{1}+\mathbf{k}_{2}-\mathbf{k}_{3}$. Assuming negligible depletion of the input waves, the amplitude of the 
outgoing wave as it emerges from the interaction region $A$ is

$$
E_{3}(\Delta \mathbf{k})=\Gamma \int_{A} g(\mathbf{r}) e^{i \Delta \mathbf{k} \cdot \mathbf{r}} d^{2} r
$$

where $\Gamma$ is a constant that depends on the amplitudes of the incoming waves and on the indices of refraction of all three waves, and $g(\mathbf{r})$ is the relevant component of $\chi^{(2)}$, coupling the three waves. Clearly, if $g$ is independent of $\mathbf{r}$ the oscillating mismatched phase averages out the output signal giving, for a rectangular $A$, a two-dimensional sinc function that tends to a delta function $\delta(\Delta \mathbf{k})$ as the interaction area $A$ tends to infinity. In general, the output signal $E_{3}(\Delta \mathbf{k})$ is proportional to the Fourier transform of $g(\mathbf{r}) \varphi_{A}(\mathbf{r})$, where $\varphi_{A}(\mathbf{r})$ is the characteristic function of the interaction area $A$ (equal to 1 if $\mathbf{r} \in A$, and 0 otherwise). We can design this Fourier transform to peak at $\Delta \mathbf{k}$ by ensuring that $\Delta \mathbf{k}$ is a vector in the reciprocal lattice of $g(\mathbf{r})$.

Consider more generally a set of $D$ nonlinear interactions of triplets of waves, coupled by $\chi^{(2)}$ as in (11), with $D$ 2-dimensional wave vectors $\Delta \mathbf{k}^{(j)}[j=1 \ldots D]$, describing their mismatches. We wish to design a nonlinear photonic crystal $g(\mathbf{r})$ that will simultaneously phase-match all $D$ interactions. If, by accident, all $D$ mismatch vectors can be expressed as integral linear combinations of two of them, $\Delta \mathbf{k}^{(1)}$ and $\Delta \mathbf{k}^{(2)}$, one can view these two vectors as the basis of a 2-dimensional reciprocal lattice. One then finds the dual real-space basis-vectors $\mathbf{a}^{(1)}$ and $\mathbf{a}^{(2)}$ whose reciprocal vectors are $\Delta \mathbf{k}^{(1)}$ and $\Delta \mathbf{k}^{(2)}$ through the condition

$$
\mathbf{a}^{(i)} \cdot \Delta \mathbf{k}^{(j)}=2 \pi \delta_{i j},
$$

and generates a real-space lattice consisting of points at all integral linear combinations of $\mathbf{a}^{(1)}$ and $\mathbf{a}^{(2)}$. The Fourier transform of a set of delta functions at these lattice positions is a set of delta function at all the reciprocal lattice positions generated by integral linear combinations of the two mismatch wave vectors, as required. This is the procedure that has been used until now $[\underline{6}, \mathbf{7}, 8,8]$.

In general, when the mismatch vectors do not all belong to a reciprocal lattice of a periodic crystal, we need to construct a quasicrystal whose Fourier transform contains all the mismatch vectors. To do so we use a generalized version of de Bruijn's dual grid method [19, 20, 21, 22] used for the construction of tiling models of quasicrystals. We begin by selecting a set of $D$ 2 -dimensional real-space vectors $\mathbf{a}^{(j)}[j=1 \ldots D]$, called tiling vectors. We use a generalization of the orthogonality condition (2), known as the Ho condition [23],

$$
\sum_{j=1}^{D} a_{\mu}^{(j)} \Delta k_{\nu}^{(j)}=2 \pi \delta_{\mu \nu} .
$$

Note that in the special case of a periodic structure the Ho condition (3) is simply the completeness relation that follows from the orthogonality condition (2) used to define the reciprocal vectors. If we were now simply to generate all points at integral linear combinations of the $D$ tiling vectors we would get the unwanted outcome of a dense filling of real space. To avoid this situation we construct the dual grid, whose topology determines which of the integral linear combination of the tiling vectors are to be included in the tiling.

To construct the dual grid we associate with each mismatch vector $\Delta \mathbf{k}^{(j)}=2 \pi \mathbf{n}^{(j)} / L_{j}$ an infinite family of parallel lines normal to the unit vector $\mathbf{n}^{(j)}$, separated by a distance $L_{j}$, and shifted from the origin (in the direction of $-\mathbf{n}^{(j)}$ ) by an amount $f_{j} L_{j}$, for some chosen set of grid shifts $0 \leq f_{j}<1$. This grid is dual to the tiling in the sense that each intersection of lines in the grid corresponds to a tile in the tiling whose edges are the tiling vectors $\mathbf{a}^{(j)}$ associated with the families of the intersecting lines. In addition, each cell in the dual grid, labeled by $D$ integers $n_{j}$, determines a vertex in the tiling at position $\sum_{j} n_{j} \mathbf{a}^{(j)}$, where $n_{j}$ is the number of lines of the $j^{\text {th }}$ family separating the cell from the origin. Only those linear combinations that correspond to cells in the dual grid are included in the tiling.

The canonical choice of tiling vectors 21, section IV.C], which is convenient for the analytical calculation of the Fourier transform, is obtained by viewing the $D$ 2dimensional mismatch vectors as $2 D$-dimensional vectors $\left(\Delta k_{\mu}^{(1)}, \ldots, \Delta k_{\mu}^{(D)}\right)[\mu=1,2]$ spanning a 2 -dimensional subspace of a $D$-dimensional vector space. One can then choose $D-2$ additional $D$-vectors $\left(q_{\mu}^{(1)}, \ldots, q_{\mu}^{(D)}\right)$ $[\mu=3 \ldots D]$, orthogonal to the first two, spanning the remaining $(D-2)$-dimensional subspace. This process adds $D-2$ components to each of the mismatch vectors extending them into the $D D$-dimensional vectors $\mathbf{K}^{(j)}=\left(\Delta \mathbf{k}^{(j)}, \mathbf{q}^{(j)}\right)$ that also span the entire $D$ dimensional vector space. One can then find the dual basis $\mathbf{A}^{(j)}=\left(\mathbf{a}^{(j)}, \mathbf{b}^{(j)}\right)$, expanded into 2-dimensional tiling vectors $\mathbf{a}^{(j)}$ and $(D-2)$-dimensional extensions $\mathbf{b}^{(j)}$, using a $D$-dimensional orthogonality condition

$$
\mathbf{A}^{(i)} \cdot \mathbf{K}^{(j)}=2 \pi \delta_{i j},
$$

which implies a $D$-dimensional completeness relation

$$
\sum_{j=1}^{D} A_{\mu}^{(j)} K_{\nu}^{(j)}=2 \pi \delta_{\mu \nu}
$$

thus satisfying the Ho condition (3). Note that the choice of the $D-2$ vectors $\left(q_{\mu}^{(1)}, \ldots, q_{\mu}^{(D)}\right)$ is not unique, but once these are chosen the tiling vectors $\mathbf{a}^{(j)}$ and their $(D-2)$ dimensional extensions $\mathbf{b}^{(j)}$ are uniquely determined by the orthogonality condition (4).

It can be shown [21, Eq. 5.5] that if $\rho(\mathbf{r})$ is a sum of delta functions, at the positions determined by the dual grid method, then its Fourier transform $\rho(\mathbf{k})$ is non-vanishing at most on the lattice of integral linear 
combinations of the $\Delta \mathbf{k}^{(j)}$. If the $\Delta \mathbf{k}^{(j)}$ are linearly independent over the integers this immediately gives us Bragg peaks at the positions required for multiple phase-matching. If the mismatch vectors are integrallydependent some of the Bragg peaks may become extinguished. To avoid this situation one may simply choose an independent subset of mismatch vectors to generate the structure, whose Fourier transform will then contain all the required Bragg peaks. Alternatively, one may refine the procedure for selecting the tiling vectors. We shall consider the latter option in greater detail elsewhere. The Fourier coefficient at $\mathbf{k}$ is given by

$$
\rho(\mathbf{k})=\frac{1}{v} \sum e^{i \mathbf{q} \cdot \mathbf{f}} \int_{W} \mathrm{~d} \mathbf{s} e^{i \mathbf{q} \cdot \mathbf{s}},
$$

where the sum - required only when the mismatch vectors are linearly-dependent over the integers - is over all $\mathbf{q}=\sum m_{j} \mathbf{q}^{(j)}$ such that $\sum m_{j} \Delta \mathbf{k}^{(j)}=\mathbf{k}$, the shift vector $\mathbf{f}=\sum f_{j} \mathbf{b}^{(j)}, W$ is the so-called $(D-2)$-dimensional window, given by the set of all points $\sum \lambda_{j} \mathbf{b}^{(j)}$ with $0 \leq \lambda_{j}<1, \mathbf{s}$ is a $(D-2)$-dimensional integration vector, and $v$ is the volume of the primitive cell of the $D$ dimensional real-space lattice generated by the vectors $\mathbf{A}^{(j)}$.

Methods for modulating the nonlinear coefficient have been developed for many $\chi^{(2)}$ materials, and in particular for ferroelectrics 24] and semiconductors 25]. Although typically the nonlinear coefficient is modulated only in one direction, the modulation methods are based on planar technologies, hence 2-dimensional modulation is possible [7]. In ferroelectrics such as $\mathrm{LiNbO}_{3}$ and $\mathrm{KTiOPO}_{4}$, the modulation is based on reversing the electrical domains in the $z$ direction of the crystal, and thus provides either a positive or negative value of the $\chi_{33}^{(2)}$ coefficient. In the following calculation, we have assumed a minimum domain size of $2 \mu \mathrm{m}$, which can be achieved using the electric field poling technique [14]. Thus the actual nonlinear crystal $g(\mathbf{r})$ is extended over a finite area $A$, and consists of positive domains of a given shape $S$, typically small circles [8] or polygons such as hexagons [7] or squares [10], positioned at the vertices of the tiling in a negative background. Using the convolution theorem, its actual Fourier transform $g(\mathbf{k})$ is then

$$
g(\mathbf{k})=\Delta \chi\left(\rho(\mathbf{k}) \otimes \int_{A} e^{i \mathbf{k} \cdot \mathbf{r}} d^{2} r\right) \int_{S} e^{i \mathbf{k} \cdot \mathbf{r}} d^{2} r,
$$

where $\Delta \chi$ is the absolute difference between the positive and negative values used for $\chi_{33}^{(2)}, \rho(\mathbf{k})$ is the sum of delta functions whose amplitudes are given in (6), and $\otimes$ is the convolution operator. For example, if $S$ is a circle of radius $R$ and $A$ a rectangle of sides $L_{x} \times L_{y}$, the Fourier transform is

$$
\begin{aligned}
g(\mathbf{k})= & g\left(k_{x}, k_{y}\right)=2 S A \Delta \chi \frac{J_{1}(k R)}{k R} \\
& \times\left(\rho(\mathbf{k}) \otimes\left[\operatorname{sinc}\left(\frac{k_{x} L_{x}}{2}\right) \operatorname{sinc}\left(\frac{k_{y} L_{y}}{2}\right)\right]\right)(8)
\end{aligned}
$$

where $k=|\mathbf{k}|$, and $J_{1}$ is the first Bessel function. There is, of course, much room for fine tuning of the design process - especially in the choice of the domain shape $S$ but we have clearly demonstrated that the required mismatch wave vectors indeed appear in the Fourier transform of the nonlinear photonic quasicrystal. Note in particular for the circle, that care must be taken in the choice of the radius $R$ such that the first zero of the Bessel function, occuring at $k R \simeq 3.8317$, does not extinguish the required Bragg peaks.

As an example for our design procedure consider the color fan, depicted in Fig. [1] whose input is a fundamental beam at frequency $\omega$ in the $\hat{x}$ direction and whose output consists of the second, third, and fourth harmonics in different directions. Note that the pair of interacting beams, in all three frequency conversion processes, $\omega$ and $2 \omega$, are travelling in the same $(\hat{x})$ direction to allow for efficient interaction throughout the full length of the photonic crystal. Using the experimental values for the dispersion in lithium niobate at $150^{\circ} \mathrm{C}$, given in Ref. 26, and taking an input beam of wavelength $\lambda=1550 \mathrm{~nm}$, the required mismatch vectors and the corresponding tiling vectors, satisfying the Ho condition (3), are given in Table II In order to keep all three mismatch vectors to be of the same magnitude we use for the first process $(\omega+\omega \rightarrow 2 \omega)$ a third order quasi-phase-matching condition, as described for example in Ref. [5. A section of the resulting photonic quasicrystal, produced with these parameters using the dual grid method, is shown in Fig. 2 Its Fourier transform is shown in Fig. 3, clearly showing Bragg peaks exactly at the required positions of the mismatch vectors.

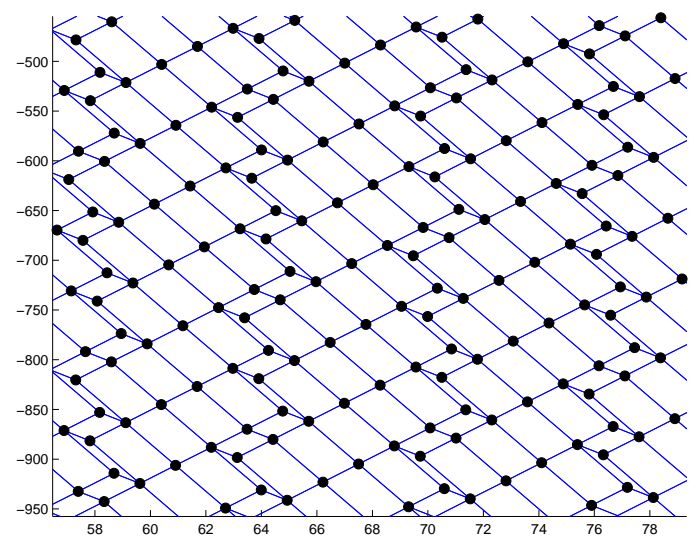

FIG. 2: Structure of the photonic quasicrystal, produced with the parameters of Table \ Scale is in $\mu m$.

The method described in this Letter enables to achieve efficient frequency mixing of any arbitrary set of input waves, in order to generate a chosen set of output waves with specified wavelengths and propagation directions. We have shown the implementation of the dualgrid method for a two-dimensional modulation of the 


\begin{tabular}{|c|c|c|c|}
\hline Mismatch vector & Magnitude in $(\mu m)^{-1}$ & Direction in radians & $g\left(\Delta \mathbf{k}^{(i)}\right)$ \\
\hline$\Delta \mathbf{k}^{(1)}=\Delta \mathbf{k}_{11 \rightarrow 2}$ & 1.0183 & 0 & 0.0404 \\
$\Delta \mathbf{k}^{(2)}=\Delta \mathbf{k}_{22 \rightarrow 4}$ & 2.8179 & 0.0456 & 0.0431 \\
$\Delta \mathbf{k}^{(3)}=\Delta \mathbf{k}_{12 \rightarrow 3}$ & 0.9530 & -0.0966 & 0.1671 \\
\hline Tiling vector & Magnitude in $\mu m$ & Direction in radians & \\
\hline $\mathbf{a}^{(1)}$ & 10.330 & -1.4801 & \\
$\mathbf{a}^{(2)}$ & 18.234 & 1.5001 & \\
$\mathbf{a}^{(3)}$ & 42.297 & -1.5289 & \\
\hline
\end{tabular}

TABLE I: Calculated mismatch vectors for the color fan and the corresponding tiling vectors. The right-hand column lists the analytically-calculated Fourier coefficients for the three processes assuming an infinite structure and circular positive domains of radius $2.9212 \mu \mathrm{m}$ in a negative background. The corresponding values obtained in a 1-dimensional periodic crystal for first and third order processes are $2 / \pi \simeq 0.6366$ and $2 / 3 \pi \simeq 0.2122$, respectively [5, p. 2635].

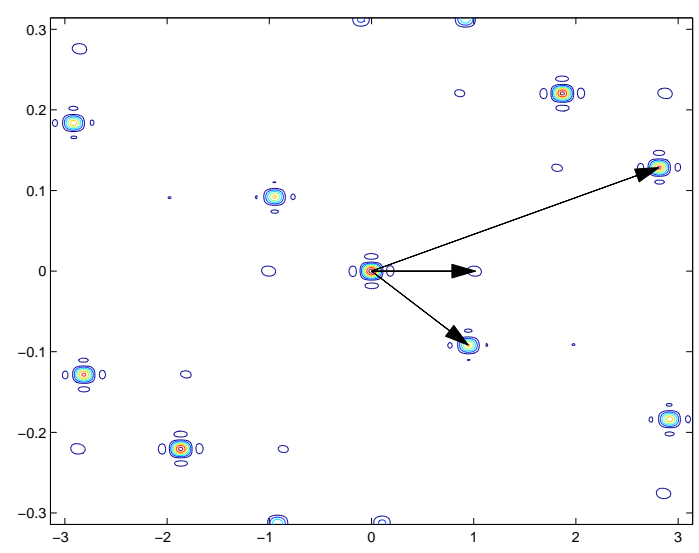

FIG. 3: Numerical Fourier transform of the crystal in Fig. 2 showing Bragg peaks at the positions of the required mismatch vectors, as indicated by arrows. Scale is in $(\mu m)^{-1}$.

nonlinear coefficient. The method is, of course, applicable in any number of dimensions. The implementation in one dimension is straightforward and requires that the wave-vectors of the interacting waves, and thus the mismatch wave-vectors, are all collinear. For example, the one-dimensional Fibonacci structure 11] can easily be generated by choosing two collinear mismatch wavevectors having a ratio of $\tau=(1+\sqrt{5}) / 2$ between them.

An interesting device that is enabled by the proposed method is a nonlinear mixer that can generate the same harmonic at several different directions. This mixer can be highly useful for cavity-enhanced nonlinear processes, such as resonant second harmonic generation and optical parametric oscillation, with the unique feature that all propagation directions in the cavity contribute to the nonlinear process. In addition to the possibility to generate multiple harmonics at multiple directions, as shown here, some interesting applications of this method can benefit from the possibility of generating large nonlinear phase shifts by cascaded nonlinear processes. These applications include generation of multicolor optical solitons, as well as all-optical deflection and switching of light [16].
This research is supported by the Israel Science Foundation through Grant No. 278/00, and by the Israeli Ministry of Science.

* Electronic address: ronlif@tau.ac.il

[1] J. A. Armstrong, N. Bloembergen, J. Ducuing, and P. S. Pershan, Phys. Rev. 127, 1918 (1962).

[2] J. A. Giordmaine, Phys. Rev. Lett. 8, 19 (1962).

[3] P. D. Maker, R. W. Terhune, M. Nisenoff, and C. M. Savage, Phys. Rev. Lett. 8, 21 (1962).

[4] N. Bloembergen and A. J. Sievers, Appl. Phys. Lett. 17, 483 (1970).

[5] M. M. Fejer, G. A. Magel, D. H. Jundt, and R. L. Byer, IEEE J. Quantum Electron. 28, 2631 (1992).

[6] V. Berger, Phys. Rev. Lett. 81, 4136 (1998).

[7] N. G. R. Broderick, G. W. Ross, H. L. Offerhaus, D. J. Richardson, and D. C. Hanna, Phys. Rev. Lett. 84, 4345 (2000).

[8] S. Saltiel and Y. S. Kivshar, Opt. Lett. 25, 1204 (2000).

[9] X.-H. Wang and B.-Y. Gu, Euro. Phys. J. B 24, 323 (2001).

[10] S. M. Russell, P. E. Powers, M. J. Missey, and K. L. Schepler, IEEE J. Quantum Elect. 37, 877 (2001).

[11] S.-N. Zhu, Y.-Y. Zhu, and N.-B. Ming, Science 278, 843 (1997).

[12] S.-N. Zhu, Y.-Y. Zhu, Y.-Q. Qin, H.-F. Wang, C.-Z. Ge, and N.-B. Ming, Phys. Rev. Lett. 78, 2752 (1997).

[13] K. Fradkin-Kashi and A. Arie, IEEE J. Quantum Electron. 35, 1649 (1999).

[14] K. Fradkin-Kashi, A. Arie, P. Urenski, and G. Rosenman, Phys. Rev. Lett. 88, 023903 (2002).

[15] Y. S. Kivshar, A. A. Sukhorukov, and S. M. Saltiel, Phys. Rev. E 60, R5056 (1999).

[16] S. M. Saltiel, A. A. Sukhorukov, and Y. S. Kivshar, Progress in Optics 44 (2004), (nlin.PS/0311013).

[17] M. Senechal, Quasicrystals and geometry (Cambridge University Press, Cambridge, 1995).

[18] R. Lifshitz, Found. Phys. 33, 1703 (2003).

[19] N. G. de Bruijn, Proc. K. Ned. Akad. Wet. Ser. A 84, 39 (1981).

[20] F. Gähler and J. Rhyner, J. Phys. A 19, 267 (1986).

[21] D. A. Rabson, T.-L. Ho, and N. D. Mermin, Acta Crystallog. A 44, 678 (1988). 
[22] D. A. Rabson, T.-L. Ho, and N. D. Mermin, Acta Crystallog. A 45, 538 (1989).

[23] T.-L. Ho, Phys. Rev. Lett. 56, 468 (1986).

[24] M. Yamada, N. Nada, M. Saitoh, and K. Watanabe, Appl. Phys. Lett. 62, 435 (1993).
[25] L. Eyers, P. J. Tourreau, T. J. Pinguet, C. B. Ebert, J. S. Harris, M. M. Fejer, L. Becouarn, B. Gerard, and E. Lallier, Appl. Phys. Lett. 79, 904 (2001).

[26] D. H. Jundt, Optics Lett. 22, 1553 (1997). 